\title{
Estudio Anatómico de la Dehiscencia Timpánica (Foramen de Huschke) en la Población Arqueológica de Tutuquén, Región del Maule, Chile
}

\author{
Anatomical Study of Tympanic Dehiscence (Foramen of Huschke) in \\ the Archaeological Population of Tutuquén, Maule Region, Chile
}

Pérez Riffo, Marcos ${ }^{1,2}$; Lindner Sanhueza, Cristian' ${ }^{1}$ \& Olave, Enrique ${ }^{3}$

PÉREZ, R.M.; LINDNER, S. C.\& OLAVE, E. Estudio anatómico de la dehiscencia timpánica (Foramen de Huschke) en la población arqueológica de Tutuquén, Región del Maule, Chile. Int. J. Morphol., 38(6):1676-1680, 2020.

RESUMEN: La dehiscencia timpánica o Foramen de Huschke (FH) se considera un rasgo craneal morfológico menor, hipostósico, no estocástico, transitorio, ubicado en laplaca timpánica del hueso temporal, cuya expresión se considera fisiológica hasta los 5 años de edad, denominándose como persistente su presencia a mayor edad. La mención de este rasgo en la literatura anatómica se presenta de manera escasa, debido a una muy baja presencia en poblaciones modernas, situación que cambia al estudiar poblaciones arqueológicas. El propósito de este estudio es describir la expresión del foramen de Huschke en una población arqueológica de Chile central. Fueron analizados 27 huesos temporales obtenidos de los restos óseos de la población del Monumento Arqueológico Cementerio Tutuquén, los cuales están depositados en el Museo Regional de Rancagua, Chile. Se evaluó presencia y ubicación del foramen, sexo, temporalidad y rango etáreo para cada muestra incluida. El FH se presentó en un 77,78 \% de los temporales, siendo 47,62 \% restos masculinos, 28,57 \% femeninos y 23,81\% de sexo indeterminado. La totalidad de los restos de niños y subadultos presentaron FH, mientras que en adultos su expresión disminuye hasta un 68,42\%. La ubicación fue un 38,10\% en cuadrante inferomedial, 33,33\% superolateral, 23,81\% superomedial, 4,71\% central. No se encontraron FH de ubicación inferolateral. El único temporal observable del período 10.000 AP (antes del presente, 1950) presentó FH, mientras que, en el período 7.000 AP se presentó en 6/7 temporales y en 14/19 temporales del período 1.000 AP. Los datos aportados complementan el conocimiento anatómico del proceso de osificación del hueso timpánico y la expresión de la dehiscencia timpánica como rasgo morfológico menor desde el estudio de poblaciones arqueológicas.

PALABRAS ClAVE: Hueso temporal; Anatomía; Cráneo; Canal auditivo; Dehiscencia timpánica.

\section{INTRODUCCIÓN}

Un rasgo discreto denominado Dehiscencia timpánica (Foramen de Huschke) corresponde a un orificio transitorio que se encuentra ubicado en la placa timpánica del hueso temporal, consecuencia del patrón de osificación bifásica que presenta dicha estructura ósea, en su desarrollo post natal, el cual progresivamente se oblitera hacia los 5 años de edad (Fricano, 2018).

Dentro del área anatómica, la mención de la dehiscencia timpánica como rasgo craneal se presenta de manera escasa, de hecho, en los textos anatómicos clásicos frecuentemente usados, solamente en uno o en dos de ellos se la describe muy someramente, y en otro se hace una alusión grafica de la misma sin acompañamiento informativo (Gray, 1918; Testut \& Latarjet, 1971). Una explicación para esto, parece ser la baja presencia de la dehiscencia timpánica en poblaciones modernas, situación que cambia al momento de estudiar poblaciones arqueológicas (Ubbelohde-Doering, 1958; Robinson, 1995; Gerszten et al., 1998; Chahuan \& Khanna, 2014; Spence et al., 2014; Rezaian et al., 2015).

El propósito de este estudio, es describir la expresión de un rasgo craneal morfológico menor, hipostósico, no estocástico denominado Foramen de Huschke o Dehiscencia timpánica, en una población arqueológica del Chile central ubicada en el Monumento Arqueológico Cementerio de Tutuquén, cuyos restos óseos pertenecen a un periodo de tiempo que se extiende desde el Holoceno Temprano hasta el Holoceno Tardío, es decir, un lapso temporal aproximado de 10.000 años AP.

\footnotetext{
${ }^{1}$ Facultad de Medicina, Universidad Católica del Maule, Talca, Chile.

${ }^{2}$ Programa Magíster en Ciencias, mención Morfología, Universidad de la Frontera, Temuco, Chile.

${ }^{3}$ Facultad de Medicina, Universidad de la Frontera, Temuco, Chile.
} 


\section{MATERIAL Y MÉTODO}

Los participantes de este estudio corresponden a huesos temporales obtenidos de los restos óseos de la población del Monumento Arqueológico Cementerio Tutuquén, Región del Maule, Chile. Fueron incluidos según los siguientes criterios: 1.- Haber sido datado y clasificado por expertos como parte de la población del Monumento Arqueológico Cementerio Tutuquén. 2.- Limpieza y mantención previa realizada por los técnicos del Laboratorio de Antropología del Consejo de Monumentos Nacionales Región del Maule. Todos los cráneos con un estado de conservación $<75 \%$ de indemnidad del hueso o placa timpánica fueron excluidos.

Restos arqueológicos de Tutuquén. El Monumento Arqueológico Cementerio Tutuquén se ubica en la vertiente occidental de la zona andina meridional sobre el valle longitudinal central entre la Cordillera de la Costa y la Cordillera de los Andes, en la confluencia de los ríos Teno y Lontué, ubicado a 2 kilómetros al oeste de la ciudad de Curicó, Región del Maule, Chile. Corresponde a un lugar que se utilizó como sitio funerario en el período comprendido entre 10.700 AP hasta 800 AP.

La colección arqueológica se ubica actualmente en el Museo Regional de Rancagua. Consta de 42 esqueletos, de los cuales 26 individuos corresponden a adultos y 16 a menores a 18 años. El conjunto de adultos se compone de 16 masculinos y 10 femeninos, mientras que los menores de 18 años se conforman por 5 lactantes ( $<2$ años) 8 niños ( $2-$ 12 años) y 3 subadultos (12 - 18 años). La datación, temporalidad y sexo de cada una de las muestras fue registrada por expertos del Monumento Arqueológico Cementerio Tutuquén (Sáez, 2008; Sáez, 2011).

Protocolo de estudio. Se identificaron los cráneos de estudio según los criterios de inclusión para la posterior selección de huesos temporales. De acuerdo al período de hallazgo se definieron tres períodos de datación: 10.000 AP; 7.000 AP y 1.000 AP. Se agruparon según sexo y estrato etáreo estimado. Se determinó presencia v/s ausencia de dehiscencia timpánica mediante visualización directa. Para determinar ubicación del FH, se trazaron 2 líneas, según la orientación del hueso timpánico, una en sentido antero - posterior y otro latero - medial, de acuerdo a los ejes de mayor longitud. Eje ventro - dorsal se considera el proceso vaginal inmediatamente medial $(2-3 \mathrm{~mm}$.) al proceso estiloides. El eje latero - medial se considera el extremo más prominente del proceso supratubal. De acuerdo a esto se determinaron cinco ubicaciones: Supero - medial, supero - lateral, ínfero - medial, ínfero - lateral y un cuadrante central definido en la confluencia de los anteriores.
En aquellos casos en que las dehiscencias timpánicas comprometieron dos o más cuadrantes, se consideró aquel ocupado en mayor proporción (Fig. 1).

Los datos fueron tabulados en MS Excel para Windows y analizados con el software IBM SPSS 10.0. Se realizó un análisis descriptivo a través de frecuencias absolutas y relativas expresadas en porcentaje.

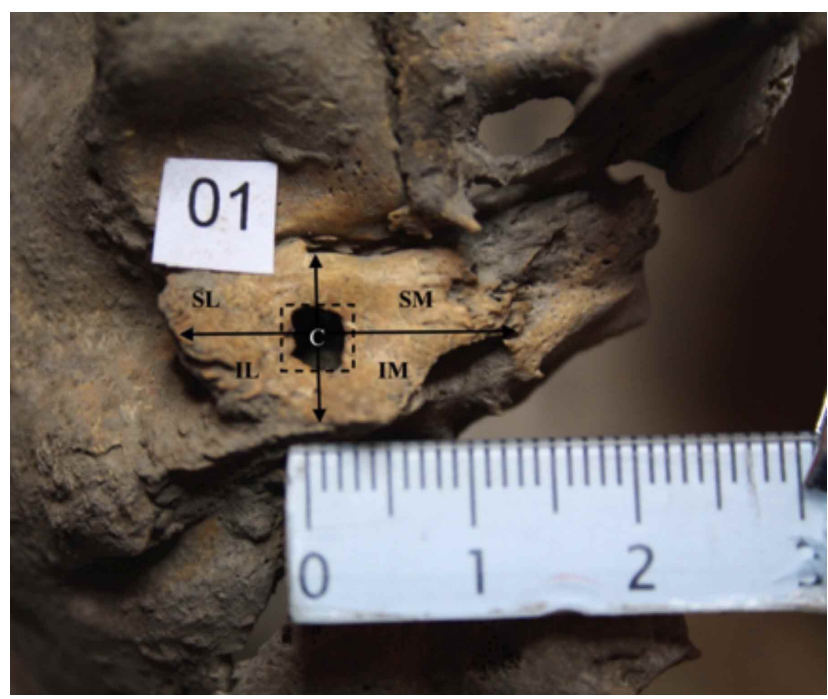

Fig.1. División del hueso timpánico en cuadrantes. (SL: Superolateral; SM: Superomedial; IL: Inferolateral; IM: Inferomedial; C: Central).

\section{RESULTADOS}

De los 42 cráneos recuperados en las excavaciones, 16 cumplieron los criterios de inclusión. A partir de éstos, se analizaron 27 huesos temporales para analizar el comportamiento de la dehiscencia timpánica. Las características generales de la muestra se resumen en Tabla I.

Se observó la presencia de FH en (21/27) 77,78 \% de los temporales observados. De éstos, (10/21) 47,62 \% fueron restos masculinos, (6/21) 28,57\% restos femeninos y (5/21) $23,81 \%$ de sexo indeterminado (Figs. 2, 3 y 4).

Un (8/21) 38,10\% de las Dehiscencias timpánicas se ubicaron en el cuadrante inferomedial; (7/21) 33,33\% superolateral; (5/21) 23,81\% superomedial y (1/21) 4,71\% central. No se encontraron FH de ubicación inferolateral. La distribución por estrato etáreo se resume en Tabla II.

En los restos pertenecientes al período $10.000 \mathrm{AP}$, el FH se presentó en el único temporal observable, mientras que en el período 7.000 AP se presentó en 6 de 7 temporales y en 14 de 19 temporales correspondientes al período 1.000 AP. 


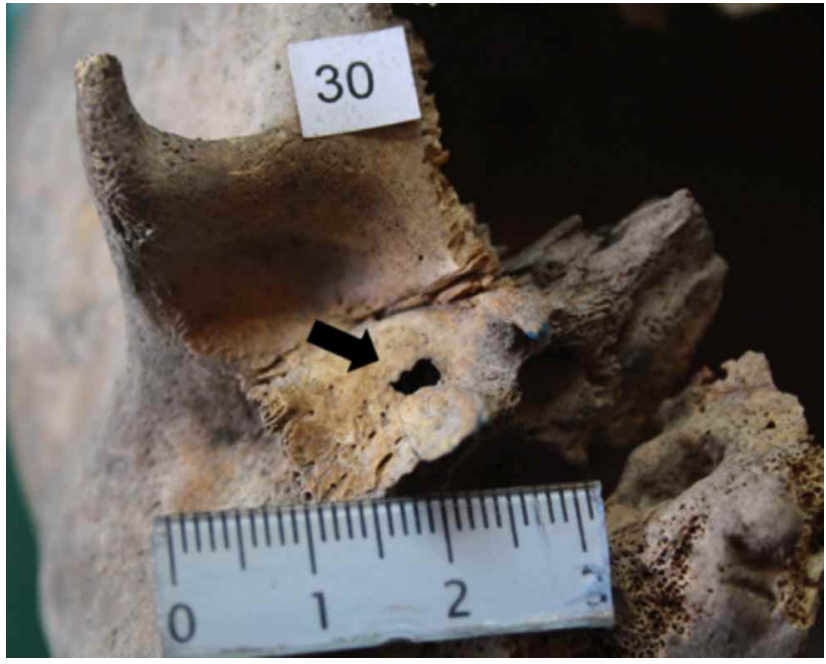

Fig. 2. Dehiscencia timpánica unilateral en individuo femenino subadulto (13-14 años de edad), datado en 1000 AP.

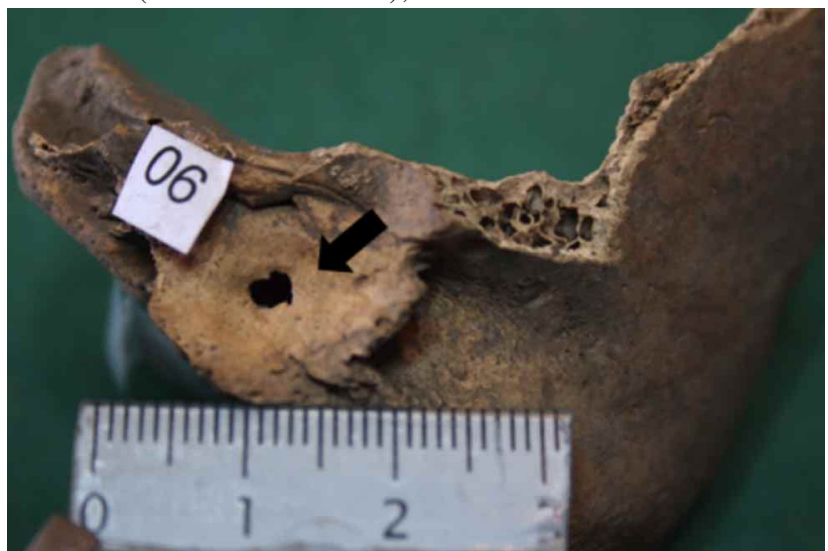

Fig. 3. Dehiscencia timpánica unilateral en individuo infantil de sexo indeterminado (5 años de edad), datado en 7000 AP.

Fig. 5. Comportamiento temporal de la Dehiscencia Timpánica en niños y adultos.

\begin{tabular}{ll}
\hline \\
\end{tabular}

\section{DISCUSIÓN}

El desarrollo post natal del hueso timpánico se lleva a cabo a partir de la formación de la porción ósea del conducto auditivo, donde comienzan a formarse dos procesos nodulares desde del margen interno del segmento medio del brazo posterior del anillo timpánico y de la proximidad del

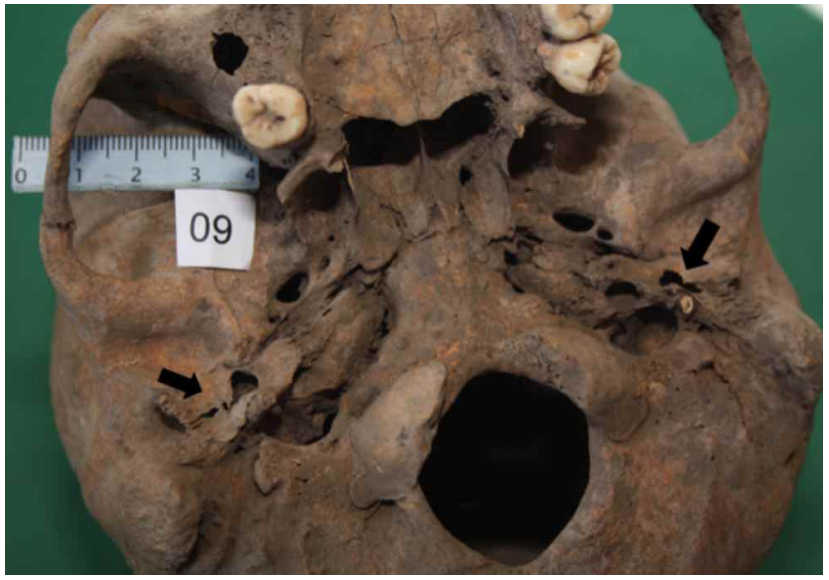

Fig. 4. Dehiscencia timpánica bilateral en individuo femenino adulto (45 años de edad) datado en $1000 \mathrm{AP}$.

Tabla I. Distribución de los cráneos por sexo, estrato etáreo y temporalidad.

\begin{tabular}{lrcc}
\hline & & Cráneos (n) & Rango Edad (años) \\
\hline \multirow{3}{*}{ Sexo } & Femenino & 8 & $13-50$ \\
& Masculino & 4 & $20-50$ \\
Estrato Etáreo & Indeterminado & 4 & $2-5$ \\
& Niño & 4 & $2-5$ \\
& Subadulto & 2 & $12-15$ \\
Temporalidad & Adulto & 10 & $20-50$ \\
& $10000 \mathrm{AP}$ & 1 & $14-15$ \\
Total & $7000 \mathrm{AP}$ & 4 & $4.5-50$ \\
& $1000 \mathrm{AP}$ & 11 & $2-50$ \\
\hline
\end{tabular}

Tabla II. Frecuencia (n, \%) de la Dehiscencia Timpánica (Foramen de Huschke) por estrato etáreo y temporalidad.

\begin{tabular}{lrccc}
\hline & & Temporale s & $\begin{array}{c}\text { Foramen } \\
\text { Huscke }\end{array}$ & $\%$ \\
\hline \multirow{4}{*}{ Estrato Etáreo } & Niño & 6 & 6 & 100 \\
& Subadulto & 2 & 2 & 100 \\
& Adulto & 19 & 13 & 68,42 \\
Temporalidad & $10000 \mathrm{AP}$ & 1 & 1 & 100 \\
& $7000 \mathrm{AP}$ & 7 & 6 & 85,71 \\
Total & $1000 \mathrm{AP}$ & 19 & 14 & 63,14 \\
& & 27 & 21 & 77,78 \\
\hline
\end{tabular}

extremo superior del brazo anterior, tubérculos timpánicos ántico y póstico de Zuckerkandl (Zuckerkandl, 1873), los que, mediante un constante crecimiento en dirección cráneo - caudal y medial - lateral logran fusionarse hacia los 18 24 meses de edad. 
Una vez realizada la fusión de estos procesos por sus vértices, quedan 2 orificios: uno superolateral respecto al punto de fusión: el futuro meato auditivo externo que comienza a crecer continuamente hacia lateral y tubularmente. Y otro inferomedial: la futura dehiscencia timpánica, qué progresivamente se osifica y queda totalmente obliterada alrededor de los 5 años de edad. En algunos casos, su permanencia más allá de la edad señalada se denomina dehiscencia timpánica persistente. (Bürkner, 1878; Heffez et al., 1989; Costa-Moreno et al., 2005; Humphrey \& Scheuer, 2006; Akcam et al., 2011; Tozoglu et al., 2012; Fricano; Ertrugul \& Keskin, 2019).

El epónimo Huschke hace honor al anatomista y embriólogo alemán Emil Huschke, quien supuestamente habría sido el primero en observarlo y describirlo en 1844 (Huschke, 1844), lo cual no es efectivo, ya que existen antecedentes de que dicha estructura fue descrita previamente por Johannes Riolanus hijo en 1648 (Riolanus, 1648). Posteriormente, Bürkner denominó a este rasgo como Dehiscencia timpánica (1878).

Al comparar la presencia de la dehiscencia timpánica con la literatura internacional se constata que nuestra serie es la que presenta la mayor expresión de dicho rasgo. Ubbelohde-Doering reportaron una presencia de 46,1 \%, Berry (1975) un 46,3\%, Gerszten et al., reportan un $50 \%$, Hanihara \& Ishida (2001) un $65 \%$ y Bilal \& Jelaka (2012) un $54 \%$. Es importante señalar que todas estas poblaciones corresponden a población peruana arqueológica y que son las que presentan mayores índices de prevalencia a nivel mundial.

Sólo se encontraron tres poblaciones no peruanas con presencia relativamente cercana: Indios fueguinos con un $56 \%$, Amerindios con un $55 \%$ e indígenas de Ontario, en quienes Spence et al. reportaron una presencia de 62,5 $\%$. Todos estos estudios se sitúan temporalmente entre los años 1.000 y 8.000 AP (Hanihara \& Ishida; Robinson).

En las demás poblaciones arqueológicas estudiadas la dehiscencia timpánica se presentó entre un 4,4\% a un $40 \%$, situándose la mayoría de ellas alrededor de un $20 \%$ (Robinson; Hanihara \& Ishida; Chahuan \& Khanna; Rezaian et al.). Dentro de la literatura internacional solamente hay dos autores que hacen mención sobre estrato etáreo: Rezaian et al., en una población iraní de 45 cráneos reportaron una prevalencia de $\mathrm{FH}$ de $4,4 \%$ sin proporcionar mayor información, mientras que Spence et al., reportaron la presencia de 5 dehiscencias; 4 de éstas en dos niños y 1 en un subadulto sin más especificación.

En la relación a presencia de la dehiscencia según sexo, sólo Bilal \& Jelaka estudiaron esta asociación en la literatura, quienes reportaron una presencia de 60,7 \% en cráneos femeninos y un 39,3 \% en masculinos. Debido a que en nuestra serie existe una presencia significativa de cráneos de sexo indeterminado, correspondiendo específicamente a cráneos infantiles, resultó dificultoso estudiar dicha asociación. Aún así, es claro que en ambos trabajos se presenta una marcada tendencia de este rasgo en el segmento femenino de la población.

Por su parte, Rezaian et al. proporcionaron la única referencia relacionada a estudio de ubicación de la dehiscencia timpánica, quienes informaron que éstas se ubican a nivel del vértice de la fisura timpanoescamosa, lo que corresponde al cuadrante superolateral.

Finalmente, en lo que respecta al comportamiento temporal de la dehiscencia timpánica según lo expuesto en la Figura 5, se observa que todas las muestras de niños y subadultos, tanto de 7.000 AP como de 1.000 AP presentan FH. Lo anterior, sería debido a que es normal encontrar este rasgo en los niños de acuerdo a la continuidad del proceso de osificación, por tanto, no se puede esperar que haya una variación inclusive en un lapso de tiempo de 6000 años, ya que es un proceso natural. A su vez, no ocurre lo mismo en los adultos, por lo que se observa una disminución de la presencia de este rasgo a lo largo de los siglos, lo cual es concordante con la información reportada en poblaciones modernas, en quienes la expresión de la dehiscencia timpánica no supera el $48 \%$ (Hanihara \& Ishida).

Existe una alta expresión de la dehiscencia timpánica en población de niños y subadultos con una ubicación preferentemente superolateral - inferomedial en la pared timpánica, mientras que, a medida que se avanza hacia la adultez su presencia va decreciendo gradualmente. Esta tendencia es transversal a todos los períodos consignados en este estudio. Nuestro bajo número muestral, del cual $25 \%$ es de sexo indeterminado y sólo un 3,70\% de temporales fue del período 10.000 AP representan las principales limitaciones de este estudio. No es posible señalar que el sexo femenino o dicho período de tiempo sean factores de predominancia de la presencia o ausencia del FH.

Los datos aportados por nuestro estudio complementan el conocimiento anatómico del proceso de osificación del hueso timpánico y la expresión de la dehiscencia timpánica como rasgo morfológico menor desde el estudio de poblaciones arqueológicas. 
PÉREZ, R. M.; LINDNER, S. C. \& OLAVE, E. Anatomical study of tympanic dehiscence (Foramen of Huschke) in the archaeological population of Tutuquén, Maule Region. Chile. Int. J. Morphol., 38(6):1676-1680, 2020.

SUMMARY: The tympanic dehiscence or Foramen of Huschke (FH) is considered a transient minor, hypostosic, nonstochastic cranial feature, located in the tympanic plate of the temporal bone. Its expression is considered physiological until the age of five, and its presence is constant after that. This characteristic is seldom mentioned in anatomical literature since it is infrequent in modern populations. However, there is a difference when archaeological populations are studied. The purpose of this study is to describe the expression of the Huschke foramen in an archaeological population of central Chile. For this analysis, 27 temporal bones were obtained from human skeletal remains of the Tutuquén Cemetery Archaeological Monument. Presence and location of the foramen, sex, time period and age range were evaluated for each sample. The FH occurred in $77.78 \%$ of the temporal bones, with $47.62 \%$ male, $28.57 \%$ female and $23.81 \%$ of undetermined sex. All children and subadults presented $\mathrm{FH}$, while in adults it decreased to $68.42 \%$. The location was $38.10 \%$ in the inferomedial quadrant, $33.33 \%$ superolateral, $23.81 \%$ superomedial, $4.71 \%$ central, and no FH was found at the inferolateral position. The only temporal bone identified in the 10,000 BP (before the present 1950) presented $\mathrm{FH}$, while in the 7,000 BP period, it presented in 6/7 temporal bones and in the $1000 \mathrm{BP}$ period, it was found in 14/ 19 temporal bones. The data provided complements anatomical knowledge of the tympanic bone ossification process, and in archaeological population studies, tympanic dehiscence presents as a lesser morphological characteristic.

KEY WORDS: Temporal bone; Anatomy; Skull; Ear canal; Tympanic dehiscence..

\section{REFERENCIAS BIBLIOGRÁFICAS}

Akcam, T.; Hidir, Y.; Ilica, A. T.; Kilic, E. \& Secimen, M. Temporomandibular joint herniation into the external ear canal through foramen of Huschke. Auris Nasus Larynx, 38(5):646-9, 2011.

Berry, A. C. Factors affecting the incidence of non-metrical skeletal variants. J. Anat., 120(Pt. 3):519-35, 1975.

Bilal, A. \& Jelaka, M. Biological sex and tympanic dehiscence. Resumen de Trabajo de Congreso. San Diego, Experimental Biology, 2012.

Bürkner, K. Kleine Kleine Beiträge zur normalen und pathologischen Anatomie des Gehörorgans. Arch. Ohrenheilkund., 13:163-95, 1878.

Chahuan, R. \& Khanna, J. Foramen of Huschke in North Indians: an anatomical study. Int. J. Res. Med. Sci., 2(2):728-32, 2014.

Costa-Moreno, R.; Chilvaquer, I.; Hayek, J. E. \& Seraidarian, P. I. Anatomoradiograph study of the persistence of Huschke's foramen. Rev. Bras. Otorrinolaringol., 71(5):676-9, 2005.

Ertrugul, S. \& Keskin, N. K. Relationship of age to foramen of Huschke and investigation of the development of spontaneous temporomandibular joint herniation. Int. J. Oral Maxillofac. Surg., 48(4):534-9, 2019.

Fricano, E. The Primate Ectotympanic Tube: Correlates of Structure, Function, and Development. Tesis de Doctorado. Baltimore, University John Hopkins, 2018.
Gerszten, P. C.; Gerszten, E. \& Allison, M. J. Diseases of the skull in preColumbian South American mummies. Neurosurgery, 42(5):1145-52, 1998.

Gray, H. Anatomy of the Human Body. $20^{\mathrm{a}}$ ed. Philadelphia, Lea \& Febiger 1918.

Hanihara, T. \& Ishida, H. Frecuency variations of discrete cranial traits in major human populations. II. Hypostotic variations. J. Anat., 198:707$25,2001$.

Heffez, L.; Anderson, D. \& Mafee, M. Developmental defects of the tympanic plate: case reports and review of the literature. J. Oral Maxillofac. Surg., 47(12):1336-40, 1989.

Humphrey, L. T. \& Scheuer, L. Age of closure of the foramen of Huschke: an osteological study. Int. J. Osteoarchaeol., 16(1):47-60, 2006.

Huschke, E. Lehre von den Eingeweiden und Sinnesorganen des Menschlichen Körpers. In: Sömmerring, S. T. (Ed.). Vom Bau des menschlichen Körpers. Umgearbeitet und Beendigt. Leipzig, Leopold Wagner, 1844.

Rezaian, J.; Namavar, M. R.; Nasab, H.; Nobari, A. \& Abedollahi, A. Foramen tympanicum or foramen of Huschke: a bioarchaeological study on human skeletons from an Iron Age Cemetery at Tabriz Kabud Mosque Zone. Iran. J. Med. Sci., 40(4):367-71, 2015.

Riolanus, J. Encheiridium Anatomicum et Pathologicum. $4^{\text {a }}$ ed. Paris, Adriani Wyngerden, 1648.

Robinson, R. Temporal Bone Variation in Australian Aborigines and Other Modern Populations: Implications for the Origins of Modern Humans. Tesis de Doctorado. Londres, University College London, 1995.

Sáez, A. Informe de Laboratorio Bioantropológico de Tutuquén. Manuscrito. Tutuquén, Consejo de Monumentos Nacionales, 2008.

Saez, S. A. Variabilidad de la morfología craneofacial en poblaciones humanas holocénicas del área andina meridional, el Cementerio arqueológico Tutuquén (7.000 - 900 AP), Región del Maule, Chile. Estrat Critic. Rev. Arqueol., 5(1):339-51, 2011.

Spence, M. W.; Williams, L. J. \& Wheelers, S. M. Death and disability in a younge phase community. Am. Antiq., 79(1):108-27, 2014.

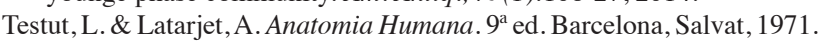

Tozoglu, U.; Caglayan, F. \& Harorli, A. Foramen tympanicum or foramen of Huschke: anatomical cone beam CT study. Dentomaxillofac. Radiol., 41(4):294-7, 2012.

Ubbelohde-Doering, H. Bericht über archäologische Feldarbeiten in Perú. J. Anthropol., 23(2-4):67-99, 1958.

Zuckerkandl,E. Zur Entwicklung des äusseren Gehörganges. Monatsschrift für Ohrenheilkunde, $\mathrm{N}^{\circ} 3,1873$.

\author{
Dirección para correspondencia: \\ Dr. Enrique Olave \\ Facultad de Medicina \\ Universidad de la Frontera \\ Temuco \\ CHILE
}

Email: enrique.olave@ufrontera.cl

Recibido : 23-06-2020

Aceptado: 30-07-2020 